\title{
Photonic analogue of Zitterbewegung in binary waveguide arrays
}

\author{
S. Longhi \\ Dipartimento di Fisica, Politecnico di Milano, Piazza L. da Vinci 32, I-20133 Milano, Italy
}

Compiled July 12, 2018

An optical analogue of Zitterbewegung (ZB), i.e. of the trembling motion of Dirac electrons caused by the interference between positive and negative energy states, is proposed for spatial beam propagation in binary waveguide arrays. In this optical system ZB is simply observable as a quiver spatial oscillatory motion of the beam center of mass around its mean trajectory. (C) 2018 Optical Society of America

OCIS codes: 230.7370, 000.2658, 050.5298

Zitterbewegung (ZB) refers to the rapid trembling motion of a free Dirac electron caused by the interference between positive and negative energy state components $[1,2]$. However, the amplitude of ZB oscillations turns out to be extremely small, of the order of the Compton wavelength, which defines the limit of electron localization. Therefore, its direct observation is unlikely, though observable consequences of ZB have been found in the response of electrons to external fields [1]. Similarly to other effects, like the Klein paradox [2], ZB has been for long time regarded as a relativistic effect rooted in the Dirac equation. However, several authors have recently shown that ZB is not unique to Dirac electrons, rather it is a generic feature of wave packet dynamics in spinor systems with certain linear dispersion relations, such as those exhibiting the so-called Dirac points (DP) that describe massless fermions [3-13]. In condensed-matter and matter-wave physics, trapped ions [3], graphene [4,5], and ultracold neutral atoms [6] have been proposed as candidate systems for a direct observation of ZB. Similarly, in the optical context it was shown that DP can occur in two-dimensional photonic crystals [7-11] or in negative-zero-positive index metamaterials [12]. Photonic analogues of ZB have been recently proposed in Refs. [11,13], in which the observation of ZB requires time-resolved measurements of pulse transmission through photonic crystal or metamaterial slabs of different thickness. In this Letter we propose a simpler optical system, consisting of a binary waveguide array, which enables an easy visualization in space of photonic ZB. The ability of mapping typical ultrafast phenomena occurring in the matter as spatial propagation of light waves in coupled waveguide structures has been successfully demonstrated in several experiments (see, e.g., [14-17]), and our proposal should thus greatly facilitate the way toward a first observation of ZB.

Let us consider propagation of monochromatic light waves at wavelength $\lambda$ in a one-dimensional optical lattice, which is described by the following scalar equation for the electric field envelope $E(x, z)$

$$
i \partial_{z} E=-\left[\lambda /\left(4 \pi n_{s}\right)\right] \partial_{x}^{2} E+(2 \pi / \lambda)\left[n_{s}-n(x)\right] E,
$$

where $n_{s}$ is the substrate refractive index and $n(x)$ is the

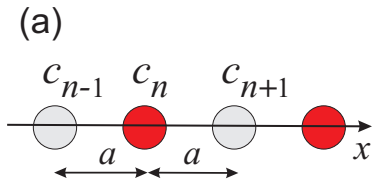

(b)
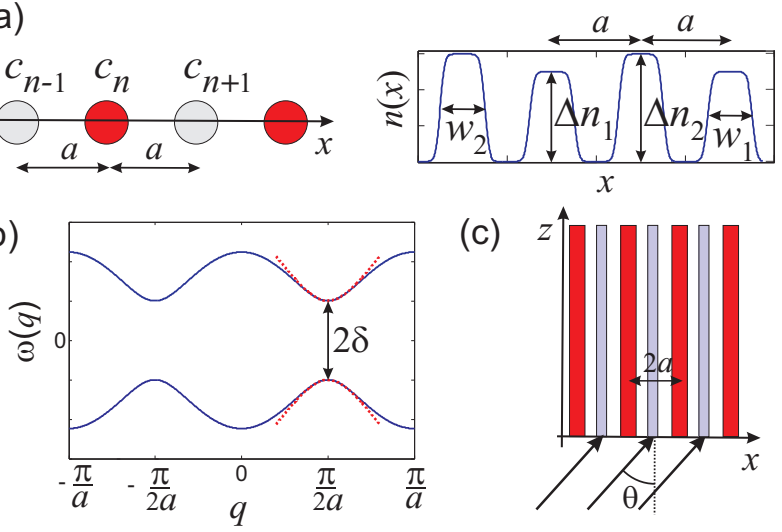

(c)

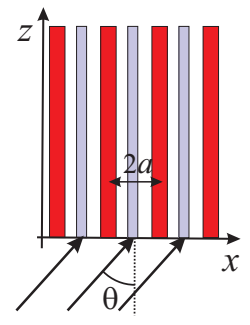

Fig. 1. (Color online) (a) Schematic of a one-dimensional binary array (left) and refractive index profile (right). (b) Dispersion curves of the first two minibands of a tightbinding binary array (solid curves), and corresponding dispersion curves of the Dirac equation (4) (dotted curves). (c) Broad-beam excitation geometry of the array at a tilting angle $\theta$.

refractive index profile of the lattice. To realize a photonic analogue of ZB, a two-band model with a dynamics described by a two-component spinor wave function is needed. Such a model can be realized by either a singlyperiodic lattice with a shallow sinusoidal refractive index profile, where the two components of the spinor wave functions are related by simple linear transformation to the amplitudes of counterpropagating waves in the lattice (see, for instance, [18]), or to a tight-binding binary superlattice composed by two interleaved sublattices A and B [see Fig.1(a)], where the spinor wave functions correspond to the occupation amplitudes in the two sublattices. Here we study ZB in the latter structure and assume a symmetric intersite coupling, which corresponds to the experimental conditions of Ref. [17]; the analysis could be extended, if needed, for the more general case of non-symmetric intersite couplings [20]. In the tight-binding approximation, light transport in the binary lattice is described by coupled-mode equations for the modal field amplitudes $c_{n}$ in the various waveg- 
uides $[17,20,21]$

$$
i\left(d c_{n} / d z\right)=-\sigma\left(c_{n+1}+c_{n-1}\right)+(-1)^{n} \delta c_{n},
$$

where $2 \delta$ and $\sigma$ are the propagation constant mismatch and the coupling rate between two adjacent waveguides of the array, respectively. The tight-binding model (2) supports two minibands, whose dispersion curves are readily obtained by the plane-wave Ansatz $c_{n}(q) \sim$ $\exp (i q n a-i \omega z)$ and read [20]

$$
\omega(q)= \pm \sqrt{\delta^{2}+4 \sigma^{2} \cos ^{2}(q a)}
$$

[see Fig.1(b)]. Let us assume that the array is excited by a broad beam (e.g. Gaussian shaped) incident onto the array at an angle close to the Bragg angle $\theta=\lambda /\left(4 n_{s} a\right)$, i.e. $E(x, 0)=G(x) \exp \left(2 \pi i \theta n_{s} x / \lambda\right)$, where $a$ is the spacing between adjacent waveguides and $G(x)$ varies slowly on the spatial scale $\sim a$ [see Fig.1(c)]. At such an incident angle, the modes in adjacent waveguides are excited with a nearly equal amplitude but with a phase difference of $\pi / 2$. After setting $c_{2 n}(z)=(-1)^{n} \psi_{1}(n, z)$ and $c_{2 n-1}=-i(-1)^{n} \psi_{2}(n, z)$, for broad beam excitation the amplitudes $\psi_{1}$ and $\psi_{2}$ vary slowly with $n$, and one can thus write $\psi_{1,2}(n \pm 1, z)=\psi_{1,2}(n, z) \pm\left(\partial \psi_{1,2} / \partial n\right)$ and consider $n \equiv \xi$ as a continuous variable rather than as an integer index. At the input plane $z=0$, the amplitudes $\psi_{1}(\xi, 0)$ and $\psi_{2}(\xi, 0)$ are proportional to $G(2 n a)$ and $G(2 n a-a) \simeq G(2 n a)$, respectively, so that one can assume $\psi_{1}(\xi, 0) \simeq \psi_{2}(\xi, 0)$ as an initial condition. Under such assumptions, from Eqs.(2) it readily follows that the two-component spinor $\psi(\xi, z)=\left(\psi_{1}, \psi_{2}\right)^{T}$ satisfies the one-dimensional Dirac equation

$$
i \partial_{z} \psi+i \sigma \alpha \partial_{\xi} \psi-\delta \beta \psi=0,
$$

where

$$
\alpha=\left(\begin{array}{ll}
0 & 1 \\
1 & 0
\end{array}\right), \beta=\left(\begin{array}{cc}
1 & 0 \\
0 & -1
\end{array}\right) .
$$

Note that $\alpha$ and $\beta$ coincide with the $\sigma_{x}$ and $\sigma_{z}$ Pauli matrices, respectively. Assuming the normalization condition $\int d \xi\left(\left|\psi_{1}\right|^{2}+\left|\psi_{2}\right|^{2}\right)=1$, after the formal change $\sigma \rightarrow c, \delta \rightarrow m c^{2} / \hbar, \xi \rightarrow x$ and $z \rightarrow t$, Eq.(4) corresponds to the one-dimensional Dirac equation for an electron of mass $m$ in absence of external fields [2]. The temporal evolution of the spinor wave function $\psi$ for the Dirac electron is therefore mapped into the spatial evolution of the modal amplitudes $\psi_{1}$ and $\psi_{2}$ in the two sublattices $\mathrm{A}$ and $\mathrm{B}$. The energy-momentum dispersion relation $\hbar \omega(k)$ of the Dirac equation (4), obtained by making the Ansatz $\psi \sim \exp (i k \xi-i \omega z)$ in Eq.(4), is composed by the two branches $\omega(k)= \pm \epsilon(k)$, corresponding to positive and negative energy states of the relativistic free electron, where $\epsilon(k)=\sqrt{\delta^{2}+\sigma^{2} k^{2}}$. Such branches reproduces the two minibands of the binary array [see Eq.(3)] near the boundary of the Brillouin zone [see Fig.1(b)], where $k=2 a q-\pi$. ZB of the Dirac electron corresponds to a rapid oscillation of the average position $\langle\xi\rangle(z)=\int d \xi \xi\left(\left|\psi_{1}\right|^{2}+\left|\psi_{2}\right|^{2}\right)$ around the classical trajectory. The usual method of understanding $\mathrm{ZB}$ in the framework of the Dirac equation is to derive equations of motion for the Heisenberg operators, and show that they oscillate in time $[1,2]$. We instead work directly in the Schrödinger picture and consider wave packet evolution in momentum space, namely we set $\psi_{1,2}(\xi, z)=\int d k \hat{\psi}_{1,2}(k, z) \exp (i k \xi)$ and calculate the spectra $\hat{\psi}_{1,2}(k, z)$ by solving Eq.(4) in momentum space. One then obtains $\hat{\psi}_{1,2}(k, z)=$ $\hat{G}(k)[\cos (\epsilon z) \mp i( \pm \sigma k+\delta) \sin (\epsilon z) / \epsilon]$, where $\hat{G}(k)=$ $(1 / 2 \pi) \int d \xi G(2 a \xi) \exp (-i k \xi)$ is the Fourier spectrum of the exciting broad input beam. The average position $\langle\xi\rangle$ is then calculated as $\langle\xi\rangle(z)=2 \pi i \int d k\left[\hat{\psi}_{1}^{*}(k) \partial_{k} \hat{\psi}_{1}(k)+\right.$ $\left.\hat{\psi}_{2}^{*}(k) \partial_{k} \hat{\psi}_{2}(k)\right]$, which yields after some algebra

$$
\begin{aligned}
\langle\xi\rangle(z) & =\langle\xi\rangle(0)+4 \pi \sigma^{3} z \int d k(k / \epsilon)^{2}|\hat{G}(k)|^{2}+ \\
& +2 \pi \sigma \delta^{2} \int d k\left(1 / \epsilon^{3}\right) \sin (2 \epsilon z)|\hat{G}(k)|^{2}
\end{aligned}
$$

The last oscillatory term in Eq.(6) is ZB, superimposed to the straight trajectory defined by the first two terms on the right hand side of Eq.(6). For $\hat{G}(k)$ spectrally narrow at around $k=0$, the frequency of $\mathrm{ZB}$ is equal to $2 \epsilon(k=0)=2 \delta$; spectral broadening of $\hat{G}(k)$ is responsible for damping of ZB. It should be noted that $\langle\xi\rangle$, calculated as the average position for the Dirac equation (4), basically reproduces the evolution of the beam center of mass $\langle n\rangle$ in real space, defined as $\langle n\rangle=$ $\left(\sum_{n} n\left|c_{n}\right|^{2}\right) / \sum_{n}\left|c_{n}\right|^{2}$. In fact, it is straightforward to show that

$$
\langle n\rangle=2\langle\xi\rangle+\frac{1}{2}-4 \pi \delta \sigma \int d k\left(k / \epsilon^{2}\right)|\hat{G}(k)|^{2} \sin ^{2}(\epsilon z) .
$$

The last term in Eq.(7) is usually negligible for a spectrum $\hat{G}(k)$ narrow at around $k=0$ (see the examples to be discussed below), so that one has $\langle n\rangle \simeq 2\langle\xi\rangle+1 / 2$. As an example, Fig.2 shows typical evolutions of field intensities $\left|c_{n}(z)\right|^{2}$ [Figs.2(a) and (c)], and corresponding behavior of beam center of mass $\langle n\rangle(z)$ [Figs.2(b) and (d), solid curves], for $\sigma=1$ and for two values of detuning $\delta$. In both cases, the array has been excited by a broad Gaussian beam $G(x)=\exp \left[-\left(x / w_{0}\right)^{2}\right]$ with $w_{0} / a=12$, and the results have been obtained by numerical integration of coupled-mode equations (2) with initial conditions $c_{n}(0)=i^{n} \exp \left[-\left(n a / w_{0}\right)^{2}\right]$. A clear trembling motion of the beam, corresponding to $\mathrm{ZB}$, is observed, with an oscillation frequency (amplitude) which increases (decreases) as $\delta$ increases, according to Eq.(6) [compare Fig.2(b) and Fig.2(d)]. A similar trembling motion would be observed for a binary lattice with asymmetric intesrite coupling [20]. Damping of ZB oscillations, which arises from the broadening of the spectrum $\hat{G}(k)$, is also visible in Figs.2(b) and (d); note also that the averaged beam path has a nonvanishing drift velocity which arises from the second term on the right hand side of Eq.(6).

We finally checked the correctness of the analysis, based 



Fig. 2. (Color online) (a) Evolution of $\left|c_{n}(z)\right|^{2}$ in a binary array excited by a broad Gaussian beam for $\sigma=1$ and $\delta=0.6$, and (b) corresponding behavior of beam trajectory $\langle n\rangle(z)$ (solid curve) as obtained by numerical analysis of Eqs.(2). The dotted curve in (b), almost overlapped with the solid curve, reproduces the behavior of $2\langle\xi\rangle(z)+1 / 2$, where $\langle\xi\rangle(z)$ is calculated according to Eq.(5). (c) and (d): same as (a) and (b), but for $\delta=1$. (a)

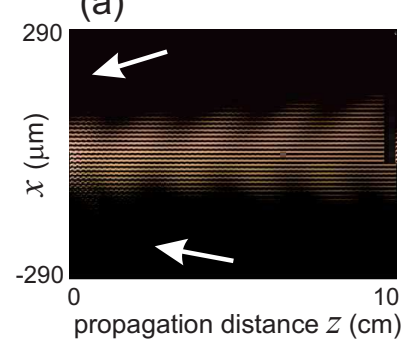

(b)

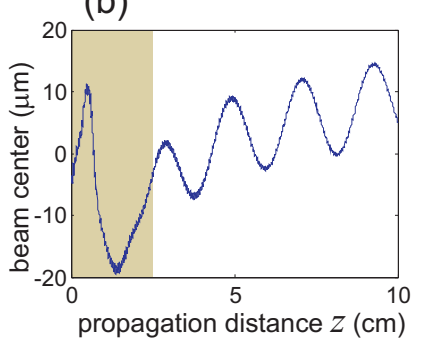

Fig. 3. (Color online) (a) Beam propagation (snapshot of $\left.|E(x, z)|^{2}\right)$ in a binary array, as obtained by numerical analysis of Eq.(1), for broad Gaussian beam excitation tilted at the Bragg angle; parameter values are given in the text. (b) Corresponding behavior of $\langle x\rangle(z)$.

on the tight-binding model (2), and the feasibility of an experimental observation of ZB by numerical simulations of the wave equation (1) for parameter values that typically apply to binary arrays realized in fused silica by femtosecond laser writing [17]. A typical numerical result is shown in Fig.3 for parameter values $\lambda=633 \mathrm{~nm}, n_{s}=1.42, a=10 \mu \mathrm{m}, w_{1}=w_{2}=3.5 \mu \mathrm{m}$, $\Delta n_{1}=0.003, \Delta n_{2}=0.00297$ and for an input Gaussian beam $E(x, 0)$ of spot size $w_{0}=80 \mu \mathrm{m}$, tilted at the Bragg angle $\theta \simeq 0.64^{\circ}$. The trembling motion of the beam as it propagates along the 10 -cm-long array is clearly visible, and should be easily observed by microscope fluorescence imaging [17]. The beam trajectory in Fig.3(b) has been computed as $\langle x\rangle(z)=\int d x x|E|^{2} / \int d x|E|^{2}$, where the integration interval is limited by the size of the numerical domain. Note that the first oscillations of $\langle x\rangle$ internal to the shaded area of Fig.3(b) do not correspond to ZB, rather they arise because of an initial beam break up and appearance of higher-order beams [as indicated by the arrows in Fig.3(a)] belonging to higher-order bands of the array. Such higher-order beams, however, refract at large angles and, after few centimeter propagation, they are no more overlapped with the main beam undergoing ZB.

In conclusion, a photonic analogue of the trembling motion of Dirac electrons has been proposed for spatial beam propagation in binary waveguide arrays. As compared to previous proposals $[11,13]$, the easy experimental visualization of beam dynamics in waveguide arrays [17] should greatly facilitate the way toward the first observation of ZB.

Author E-mail address: longhi@fisi.polimi.it

\section{References}

1. K. Huang, Am. Phys. J. 20, 479 (1952).

2. W. Greiner, Relativistic Quantum Mechanics (SpringerVerlag, Berlin, 1990).

3. L. Lamata, J. León, T. Schätz, and E. Solano, Phys. Rev. Lett. 98253005 (2007).

4. J. Cserti and G. David, Phys. Rev. B 74, 172305 (2006).

5. T. M. Rusin and W. Zawadzki, Phys. Rev. B 76, 195439 (2007).

6. J.Y. Vaishnav and C.W. Clark, Phys. Rev. Lett. 100, 153002 (2008).

7. F.D.M. Haldane and S. Raghu, Phys.Rev.Lett. 100, 013904 (2008).

8. R.A. Sepkhanov, Ya. B. Bazaliy, and C.W.J. Beenakker, Phys. Rev. A 75, 063813 (2007).

9. O. Peleg, G. Bartal, B. Freedman, O. Manela, M. Segev, and D.N. Christodoulides, Phys. Rev. Lett. 98, 103901 (2007).

10. O. Bahat-Treidel, O. Peleg, and M. Segev, Opt. Lett. 33, 2251 (2008).

11. X. Zhang, Phys. Rev. Lett. 100, 113903 (2008).

12. L.-G. Wang, Z.-G. Wang, J.-X. Zhang, and S.-Y. Zhu, Opt. Lett. 34, 1510 (2009).

13. L.-G. Wang, Z.-G. Wang, and S.-Y. Zhu, EPL 86, 47008 (2009).

14. H. Trompeter, T. Pertsch, F. Lederer, D. Michaelis, U. Streppel, A. Bräuer, and U. Peschel, Phys. Rev. Lett. 96, 023901 (2006).

15. S. Longhi, Laser \& Photon. Rev. 3, 243261 (2009).

16. G. Della Valle, M. Savoini, M. Ornigotti, P. Laporta, V. Foglietti, M. Finazzi, L. Duo, and S. Longhi, Phys. Rev. Lett. 102, 180402 (2009).

17. F. Dreisow, A. Szameit, M. Heinrich, T. Pertsch, S. Nolte, A. Tünnermann, and S. Longhi, Phys. Rev. Lett. 102, 076802 (2009).

18. J. Feng, Opt. Lett. 18, 1302 (1993).

19. A.A. Sukhorukov and Y.S. Kivshar, Phys. Rev. Lett. 91, 113902 (2003).

20. A.A. Sukhorukov and Y.S. Kivshar, Opt. Lett. 27, 2112 (2002).

21. R. Morandotti, D. Mandelik, Y. Silberberg, J.S. Aitchison, M. Sorel, D.N. Christodoulides, A.A. Sukhorukov, and Y.S. Kivshar, Opt. Lett. 29, 2890 (2004). 